\title{
Graft-versus-Host Unidirectional Mismatch
}

National Cancer Institute

\section{Source}

National Cancer Institute. Graft-versus-Host Unidirectional Mismatch. NCI Thesaurus. Code C126301.

The state when a recipient of a hematopoietic stem cell transplantation is not fully matched with their unrelated adult volunteer donor for HLA-A, HLA-B, HLA-C, and HLADRB1 and the heterozygous recipient is receiving a graft from a homozygous donor. 